\title{
A RESONANCE CALCULATION METHOD USING ENERGY EXPANSION BASED ON A REDUCED ORDER MODEL: USE OF ULTRA- FINE GROUP SPECTRUM CALCULATION AND APPLICATION TO HETEROGENEOUS GEOMETRY
}

\author{
Ryoichi Kondo $^{1}$, Tomohiro Endo ${ }^{1}$, Akio Yamamoto ${ }^{1}$, Satoshi Takeda ${ }^{2}$, Hiroki Koike ${ }^{3}$, \\ Kazuya Yamaji ${ }^{3}$, Koichi Ieyama ${ }^{3}$, and Daisuke Sato $^{3}$ \\ ${ }^{1}$ Nagoya University \\ Furo-cho, Chikusa-ku, Nagoya, Japan, 464-8603 \\ ${ }^{2}$ Osaka University \\ 2-1 Yamadaoka, Suita-shi, Osaka, Japan, 565-0871 \\ ${ }^{3}$ Mitsubishi Heavy Industries, Ltd. \\ 1-1-1 Wadasaki-Cho, Hyogo-ku, Kobe, Japan, 652-8585 \\ r-kondo@fermi.energy.nagoya-u.ac.jp, \{t-endo, a-yamamoto\}@energy.nagoya-u.ac.jp, \\ takeda@see.eng.osaka-u.ac.jp, \{hiroki_koike, kazuya_yamaji, koichi_ieyama, \\ d_sato\}@mhi.co.jp
}

\begin{abstract}
A Resonance calculation using energy Spectral Expansion (RSE) method has been recently proposed in order to efficiently treat complicated heterogeneous geometry and resonance interference effect. In the RSE method, ultra-fine group spectra are generated from ultra-fine group calculations in homogeneous geometry, and the spectra are expanded by the orthogonal basis on energy based on the singular value decomposition. Then the transport calculation for expansion coefficients is numerically performed, and the ultra-fine group spectra in the target heterogeneous regions are reconstructed by the expansion coefficients and the orthogonal basis. In this study, the RSE method is applied to multi-cell geometries including UO2, MOX and water cells, in which the resonance interference effect between UO2 and MOX fuel cells appears. The validity of the RSE method is confirmed through comparison with the reference effective multi-group cross sections obtained from the direct ultra-fine group calculation in the target heterogeneous geometry.
\end{abstract}

KEYWORDS: Resonance calculation, reduced order model, low-rank approximation, ultra-fine group spectra

\section{INTRODUCTION}

The resonance calculation is one of the most difficult and stiff parts in core analysis and can dominate the prediction accuracy of the core analysis. The equivalence theory, the ultra-fine group theory, and the subgroup theory (or combination of these methods) have been conventionally used [1]. These methods have advantages and disadvantages from the viewpoint of computational cost and accuracy. The 
equivalence theory is computationally efficient but its accuracy and geometric flexibility are limited. The ultra-fine group theory is accurate and flexible but requires large computational resources and prohibitive for a large configuration such as a whole fuel assembly or a full core. The subgroup theory can provide an efficient solution while maintaining reasonable accuracy and geometric flexibility but the treatment of the resonance interference effect among different nuclides and regions is limited.

The RSE method has been recently proposed by the authors as an alternative resonance calculation method [2]. In this approach, continuous (pointwise) neutron energy spectra in heterogeneous regions are expanded by orthogonal bases on continuous energy. The singular value decomposition (SVD) and the low-rank approximation (LRA) [3-4] are used to construct orthogonal basis on continuous energy from the calculation results of ultra-fine group spectrum obtained in a simple geometry (e.g. homogeneous geometry for typical materials and background cross sections). The proposed method has several advantages over conventional methods in theory. For example, the resonance interference effect among different heterogeneous regions can be explicitly considered.

In the present study, the RSE method is applied to $2 \times 2$ multi-cell geometries including UO2, MOX, and water cells, in which the resonance interference effect appears. Effective multi-group cross sections obtained from the RSE method are compared with those obtained from the direct ultra-fine group calculations in the target heterogeneous geometry, and accuracy of the proposed method is confirmed.

Section 2 briefly reviews the theory of the RSE method. Section 3 describes calculation conditions followed by numerical results in Section 4. Concluding remarks are provided in Section 5.

\section{THEORY}

The transport equation assuming isotropic scattering and neutron sources is:

$$
\mathbf{\Omega} \cdot \nabla \Psi(\mathbf{r}, \mathbf{\Omega}, E)+\Sigma_{t}(\mathbf{r}, E) \Psi(\mathbf{r}, \mathbf{\Omega}, E)=\frac{1}{4 \pi} Q(\mathbf{r}, E)
$$

Consider a multi-group energy range (energy range for multi-group $g, 1 \leq g \leq \mathrm{G}$ )):

$$
\boldsymbol{\Omega} \cdot \nabla \Psi\left(\mathbf{r}, \mathbf{\Omega}, E_{g}\right)+\Sigma_{t}\left(\mathbf{r}, E_{g}\right) \Psi\left(\mathbf{r}, \mathbf{\Omega}, E_{g}\right)=\frac{1}{4 \pi} Q\left(\mathbf{r}, E_{g}\right) .
$$

Angular flux, scalar flux, and neutron source are expanded by the orthogonal bases on energy:

$$
\begin{gathered}
\Psi\left(\mathbf{r}, \mathbf{\Omega}, E_{g}\right)=\sum_{i=1}^{N} f_{i, g}\left(E_{g}\right) \Psi_{i, g}(\mathbf{r}, \mathbf{\Omega}), \\
\phi\left(\mathbf{r}, E_{g}\right)=\sum_{i=1}^{N} f_{i, g}\left(E_{g}\right) \phi_{i, g}(\mathbf{r}), \\
Q\left(\mathbf{r}, E_{g}\right)=\sum_{i=1}^{N} f_{i, g}\left(E_{g}\right) Q_{i, g}(\mathbf{r}),
\end{gathered}
$$

where the orthogonal property of the bases is:

$$
\int_{\Delta E_{g}} f_{i, g}\left(E_{g}\right) f_{j, g}\left(E_{g}\right) d E_{g}=\delta_{i j}
$$


By substituting Eqs.(3)-(5) into Eq.(2), and multiplying $f_{n, g}\left(E_{g}\right)$ to the both sides of Eq.(2) and performing energy integration for $\Delta E_{g}$, we have:

where

$$
\boldsymbol{\Omega} \cdot \nabla \Psi_{n, g}(\mathbf{r}, \mathbf{\Omega})+\sum_{i=1}^{N} \Sigma_{t, g, n, i}(\mathbf{r}) \Psi_{i, g}(\mathbf{r}, \mathbf{\Omega})=\frac{1}{4 \pi} Q_{n, g}(\mathbf{r}),
$$

$$
\Sigma_{t, g, n, i}(\mathbf{r})=\int_{\Delta E_{g}} f_{n, g}\left(E_{g}\right) \Sigma_{t}\left(\mathbf{r}, E_{g}\right) f_{i, g}\left(E_{g}\right) d E_{g} .
$$

In the RSE method, Eq.(7) is solved with the cross sections defined by Eq.(8). Note that the neutron source in Eq.(7) is directly obtained by an ultra-fine group calculation in the target heterogeneous geometries in this study. Treatment of the scattering source will be one of the future tasks.

In principle, any complete orthogonal basis can be used for energy spectra and various orthogonal bases are used in the previous works [5-9]. Since the number of expansions to achieve sufficient accuracy will increase when an inappropriate basis is used, one of the key points of the RSE method is the selection of orthogonal basis.

In order to make efficient orthogonal basis that reproduces various neutron spectra in heterogeneous regions, SVD, LRA and ultra-fine group calculations in simple geometries are used, which is sometimes called reduced order model (ROM). The ultra-fine group calculations are carried out in homogeneous geometry with various background cross sections in this study. In other words, neutron spectra in heterogeneous regions are expressed by the linear combination of orthogonal basis obtained in a homogeneous geometry. This concept is similar to the equivalence theory with the multi-term rational approximation. Note that computational cost of the ultra-fine group calculation in the RSE method is not large since the calculation is carried out in simple geometries such as homogeneous geometry. Therefore, the computational cost of the RSE method can be smaller than that of the conventional method using ultra-fine group calculation in heterogeneous geometry.

\section{CALCUlations}

\subsection{Calculation Flow}

The following calculation flow is used in the present study:

(1) Perform ultra-fine group slowing down calculations in the homogeneous geometry with various background cross sections. This calculation is similar to the current practice to generate microscopic effective cross sections using a nuclear data processing code (e.g., NJOY [10]), but slowing down calculations are carried out for different materials (e.g., $\mathrm{UO}_{2}, \mathrm{MOX}, \mathrm{H}_{2} \mathrm{O}$ ) appeared in heterogeneous geometry rather than a material of single pure isotope. Background cross sections are chosen to cover the wide range of self-shielding conditions from the fully shielded (very small background cross section) to the fully diluted (very large background cross section) conditions.

(2) Repeat Step (1) for different materials used in the calculation geometry. If a material contains no resonance nuclide, the spectrum calculation for this material may be skipped since its spectrum is covered by the fully diluted condition of other materials.

(3) Collect ultra-fine group spectra obtained by Step (2) and perform SVD and LRA to obtain orthogonal basis. SVD and LRA are independently applied for each multi-energy group. Thus, the orthogonal bases are different among the multi-energy group. However, the same orthogonal basis is used for an energy group throughout a heterogeneous geometry.

(4) Generate $\Sigma_{t, g, n, i}$ using Eq.(8). 
(5) Perform MOC transport calculation using Eq.(7). Note again that the scattering term in the right-hand side of Eq.(5) is not explicitly treated in the present study. The actual treatment is described later. Equation (7) consists of simultaneous differential equations on the angular flux moment $\Psi_{i, g}$. In the present study, a direct numerical solution using the matrix exponential (instead of the conventional MOC solution using the exponential function for scalar) is adopted. An in-house MOC transport code that solves Eq.(7) is newly developed and used in the present study. The ray trace information from the GENESIS code is used in the in-house code.

(6) Once $\Psi_{i, g}$ is obtained in Step (5), ultra-fine group angular flux is reconstructed by Eq.(3).

(7) Effective microscopic cross sections for each energy group are calculated by scalar flux (obtained by solid angle integration of angular flux in Step (6)) and ultra-fine group microscopic cross sections.

(8) In order to obtain a reference solution, the ultra-fine group slowing down calculation is carried out in the heterogeneous geometry using the conventional MOC.

(9) Effective cross sections obtained in Step (7) are compared with the reference obtained in Step (8).

In the present study, the right-hand side of Eq.(7) is taken from the ultra-fine group calculation in Step (8) for consistent treatment of scattering source between the present method and the reference calculation. Then the right-hand side of Eq.(7) is expressed as:

$$
\frac{1}{4 \pi} Q_{n, g}(\mathbf{r})=q_{n, g}(\mathbf{r})=\int_{\Delta E_{g}} f_{n, g}\left(E_{g}\right) q\left(\mathbf{r}, E_{g}\right) d E_{g}
$$

where $q\left(\mathbf{r}, E_{g}\right)$ is the pointwise neutron source obtained by ultra-fine group calculation in Step (8). For a practical implementation of the RSE method, the ultra-fine group spectrum can be obtained by slowing down calculation in each heterogeneous geometry. This implementation will be tested in the future.

\subsection{Calculation Conditions}

The multi-cell geometries and the unit pin-cell geometry used in the present study are shown in Fig. 1 and Table I, respectively. Cells in Fig. 1-(a), which have the same composition are numbered for identification. Since UO2 and MOX fuel cells are adjacent in Fig. 1-(b), the resonance interference effect between two fuel cells is observed. Composition of each material is shown in Table II. The temperature of all material is $600 \mathrm{~K}$. The reflective boundary condition is assumed for all cases.

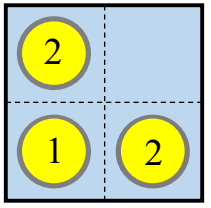

(a)

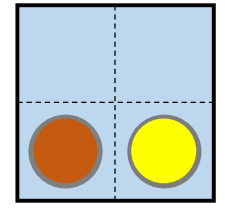

(b)

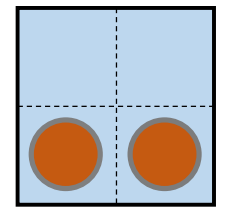

(c)

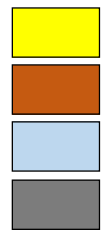

$\mathrm{UO} 2$

MOX

Moderator

Cladding

Figure 1. Multi-cell geometries.

Table I. Dimensions of pin cell

\begin{tabular}{c|c}
\hline Parameter & Value $[\mathrm{cm}]$ \\
\hline \hline Pellet radius & 0.4095 \\
\hline Cladding radius & 0.4750 \\
\hline Cell pitch & 1.2600 \\
\hline
\end{tabular}


Table II. Compositions of materials

\begin{tabular}{|c|c|c|c|c|c|}
\hline Material & Nuclide & $\begin{array}{c}\text { Number density } \\
{[1 / \text { barn } / \mathrm{cm}]}\end{array}$ & Material & Nuclide & $\begin{array}{c}\text { Number density } \\
{[1 / \mathrm{barn} / \mathrm{cm}]}\end{array}$ \\
\hline \multirow{3}{*}{$\begin{array}{c}\text { Fuel } \\
\left(\mathrm{UO}_{2}\right)\end{array}$} & ${ }^{16} \mathrm{O}$ & 4.68618E-02 & \multirow{10}{*}{$\begin{array}{c}\text { Fuel } \\
\text { (MOX) }\end{array}$} & ${ }^{16} \mathrm{O}$ & 4.59163E-02 \\
\hline & ${ }^{235} \mathrm{U}$ & $1.13836 \mathrm{E}-03$ & & ${ }^{235} \mathrm{U}$ & $4.16028 \mathrm{E}-05$ \\
\hline & ${ }^{238} \mathrm{U}$ & $2.22834 \mathrm{E}-02$ & & ${ }^{238} \mathrm{U}$ & $2.04972 \mathrm{E}-02$ \\
\hline \multirow{2}{*}{$\begin{array}{l}\text { Moderator } \\
\left(\mathrm{H}_{2} \mathrm{O}\right)\end{array}$} & ${ }^{1} \mathrm{H}$ & $4.41629 \mathrm{E}-02$ & & ${ }^{238} \mathrm{Pu}$ & 4.87049E-05 \\
\hline & ${ }^{16} \mathrm{O}$ & $2.20897 \mathrm{E}-02$ & & ${ }^{239} \mathrm{Pu}$ & $1.40652 \mathrm{E}-03$ \\
\hline \multirow{5}{*}{$\begin{array}{l}\text { Cladding } \\
\text { (Zirconium) }\end{array}$} & ${ }^{90} \mathrm{Zr}$ & $1.96348 \mathrm{E}-02$ & & ${ }^{240} \mathrm{Pu}$ & $5.55429 \mathrm{E}-04$ \\
\hline & ${ }^{91} \mathrm{Zr}$ & $4.28188 \mathrm{E}-03$ & & ${ }^{241} \mathrm{Pu}$ & $2.40495 \mathrm{E}-04$ \\
\hline & ${ }^{92} \mathrm{Zr}$ & $6.54495 \mathrm{E}-03$ & & ${ }^{242} \mathrm{Pu}$ & $1.19746 \mathrm{E}-04$ \\
\hline & ${ }^{94} \mathrm{Zr}$ & $6.63272 \mathrm{E}-03$ & & ${ }^{241} \mathrm{Am}$ & $4.80973 \mathrm{E}-05$ \\
\hline & ${ }^{96} \mathrm{Zr}$ & $1.06856 \mathrm{E}-03$ & & & \\
\hline
\end{tabular}

For the pointwise cross sections used in the present method, the ACE formatted cross sections, which are generated by the FRENDY code [11] using JENDL-4.0 [12], are adopted. The generated ACE cross sections are also used for ultra-fine group (UFG) reference calculations by MOC. The GENESIS code [13] is used as the MOC transport solver for the ultra-fine group calculation in the multi-cell geometries. The following calculation conditions are used in the GENESIS code:

-Number of polar angles: 6 for $\pi$ using the TY quadrature set [14]

-Number of azimuthal angles: 32 for $2 \pi$

-Ray trace width: $0.05 \mathrm{~cm}$

-Convergence criterion for scalar flux: $10^{-5}$

The energy group structure for ultra-fine group calculation is shown in Table III. For the multi-group structure, the XMAS 172 group structure is adopted. Namely, the orthogonal basis is independently generated in each of the 172 groups.

Table III. Energy group structure for ultra-fine group calculation

\begin{tabular}{cc}
\hline $\begin{array}{c}\text { Upper energy boundary } \\
{[\mathrm{eV}]}\end{array}$ & $\begin{array}{c}\text { Number of divisions } \\
\text { (Equal division for lethargy) }\end{array}$ \\
\hline $20,000,000$ & 10,000 \\
\hline 52,475 & 56,000 \\
\hline 9118.8 & 12,000 \\
\hline 4307.4 & 12,000 \\
\hline 961.12 & 8,000 \\
\hline 130.07 & 12,000 \\
\hline 0.32242 & 10,000 \\
\hline Lower energy boundary: $0.00001 \mathrm{eV}$
\end{tabular}

The number of ultra-fine group spectra used in the Step (3) of the calculation flow is 32, which comes from the numbers of background cross sections for $\mathrm{UO}_{2}$ and $\mathrm{MOX}$ (11 points for each from $10^{-3}$ to $10^{10}$ barn), Cladding ( 9 points from $5 \times 10^{1}$ to $5 \times 10^{6}$ barn) and $\mathrm{H}_{2} \mathrm{O}$ (1 point, $10^{10}$ barn). A maximum number of the orthogonal bases is limited by the smaller of the number of ultra-fine group within a multi-group or 
the total number of background cross sections (32). Since the minimum number of ultra-fine energy group within a multi group is 17,16 (the minimum number -1) is used as the maximum number of the orthogonal bases in the present study.

\section{NUMERICAL RESULTS}

Relative errors of the effective microscopic total cross sections are calculated. The relative error is defined by (RSE - UFG)/UFG. Table IV and Table V show the maximum absolute relative error for resonance nuclides of Fig. 1-(a), (b), and (c) in the entire resolved resonance energy range. The number of bases is fixed to 16 in Table IV and Table V. These table indicates that the RSE method accurately reproduces reference effective cross section of any nuclides.

Next, the impact of the number of bases used in the RSE method is discussed. Group $88(6.16 \mathrm{eV}-7.52$ $\mathrm{eV}$ ) in the XMAS 172 group structure is considered since a large resonance of U-238 exists in this group. Figure 2 and Fig. 3 indicate that errors are rapidly decreased as the expansion order increases and are almost converged by four orthogonal bases for Fig. 1-(a) and eight for Fig. 1-(b), respectively.

Finally, the capability to treat the resonance interference effect is discussed. In order to confirm the effect, the UFG calculations using MOC are carried out for the two geometries Fig. 1-(b) and Fig. 1-(c). The differences of the effective total cross sections for U-235, U-238, Pu-239, and Pu-240 in MOX cell obtained by UFG with MOC are shown in Fig. 4. The difference is defined by $(c-b) / b$. The energy range of Fig. 4 includes major resolved resonance of U-235 and U-238 $(1 \mathrm{eV}-1000 \mathrm{eV})$. Figure 4 shows that the effective total cross sections in (b) and (c) are different due to different material arrangement and resonance interference effect. The error of effective total cross sections in Fig. 1-(b) and (c) are shown in Fig. 5 and Fig. 6, respectively. Error of each multi group is very small, and these results indicate that the RSE method can accurately treat the resonance interference effect. Namely, the neutron spectra in the heterogeneous geometry in which the resonance interference effects appear, are expressed by the linear combination of orthogonal basis obtained in the homogeneous geometry in the present study.

Table IV. The maximum relative errors of effective total cross sections calculated by the RSE method in Fig .1-(a)

\begin{tabular}{|c|c|c|c|}
\hline Material & Nuclide & Maximum error [\%] & Multi group \\
\hline \multirow{5}{*}{$\begin{array}{l}\text { Cladding } \\
\text { (in cell 1) }\end{array}$} & ${ }^{90} \mathrm{Zr}$ & 0.90 & 49 \\
\hline & ${ }^{91} \mathrm{Zr}$ & 1.29 & 49 \\
\hline & ${ }^{92} \mathrm{Zr}$ & 1.15 & 51 \\
\hline & ${ }^{94} \mathrm{Zr}$ & 0.25 & 47 \\
\hline & ${ }^{96} \mathrm{Zr}$ & 2.76 & 63 \\
\hline \multirow{5}{*}{$\begin{array}{l}\text { Cladding } \\
\text { (in cell 2) }\end{array}$} & ${ }^{90} \mathrm{Zr}$ & 0.80 & 49 \\
\hline & ${ }^{91} \mathrm{Zr}$ & 1.20 & 49 \\
\hline & ${ }^{92} \mathrm{Zr}$ & 1.00 & 51 \\
\hline & ${ }^{94} \mathrm{Zr}$ & 0.22 & 47 \\
\hline & ${ }^{96} \mathrm{Zr}$ & 2.38 & 63 \\
\hline \multirow{2}{*}{$\begin{array}{c}\mathrm{UO} 2 \\
\text { (in cell 1) }\end{array}$} & ${ }^{235} \mathrm{U}$ & 0.35 & 75 \\
\hline & ${ }^{238} \mathrm{U}$ & 0.41 & 64 \\
\hline \multirow{2}{*}{$\begin{array}{c}\mathrm{UO} 2 \\
\text { (in cell 2) }\end{array}$} & ${ }^{235} \mathrm{U}$ & 0.39 & 75 \\
\hline & ${ }^{238} \mathrm{U}$ & 0.45 & 64 \\
\hline
\end{tabular}


Table V. The maximum relative errors of effective total cross sections calculated by the RSE method in Fig.1-(b)

\begin{tabular}{|c|c|c|c|}
\hline Material & Nuclide & Maximum error [\%] & Multi group \\
\hline \multirow{5}{*}{$\begin{array}{c}\text { Cladding } \\
\text { (in MOX cell) }\end{array}$} & ${ }^{90} \mathrm{Zr}$ & 0.35 & 49 \\
\hline & ${ }^{91} \mathrm{Zr}$ & 0.91 & 49 \\
\hline & ${ }^{92} \mathrm{Zr}$ & 0.73 & 49 \\
\hline & ${ }^{94} \mathrm{Zr}$ & 0.22 & 47 \\
\hline & ${ }^{96} \mathrm{Zr}$ & 1.46 & 63 \\
\hline \multirow{5}{*}{$\begin{array}{c}\text { Cladding } \\
\text { (in UO2 cell) }\end{array}$} & ${ }^{90} \mathrm{Zr}$ & 0.39 & 49 \\
\hline & ${ }^{91} \mathrm{Zr}$ & 0.95 & 49 \\
\hline & ${ }^{92} \mathrm{Zr}$ & 0.76 & 49 \\
\hline & ${ }^{94} \mathrm{Zr}$ & 0.22 & 47 \\
\hline & ${ }^{96} \mathrm{Zr}$ & 1.60 & 63 \\
\hline \multirow{8}{*}{ MOX } & ${ }^{235} \mathrm{U}$ & 0.23 & 69 \\
\hline & ${ }^{238} \mathrm{U}$ & 0.64 & 64 \\
\hline & ${ }^{238} \mathrm{Pu}$ & 0.32 & 66 \\
\hline & ${ }^{239} \mathrm{Pu}$ & 0.26 & 63 \\
\hline & ${ }^{240} \mathrm{Pu}$ & 1.25 & 63 \\
\hline & ${ }^{241} \mathrm{Pu}$ & 0.27 & 64 \\
\hline & ${ }^{242} \mathrm{Pu}$ & 1.28 & 69 \\
\hline & ${ }^{241} \mathrm{Am}$ & 0.63 & 69 \\
\hline \multirow{2}{*}{$\mathrm{UO} 2$} & ${ }^{235} \mathrm{U}$ & 0.23 & 69 \\
\hline & ${ }^{238} \mathrm{U}$ & 0.62 & 64 \\
\hline
\end{tabular}
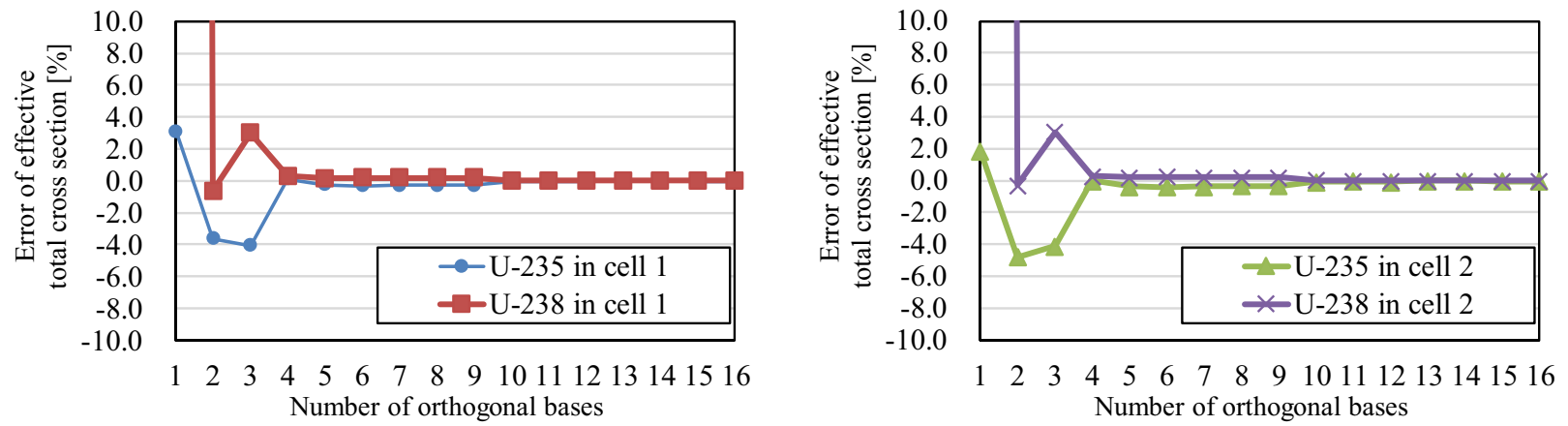

Figure 2. Comparison of effective total cross section in group 88 for various number of orthogonal bases in Fig. 1-(a). 

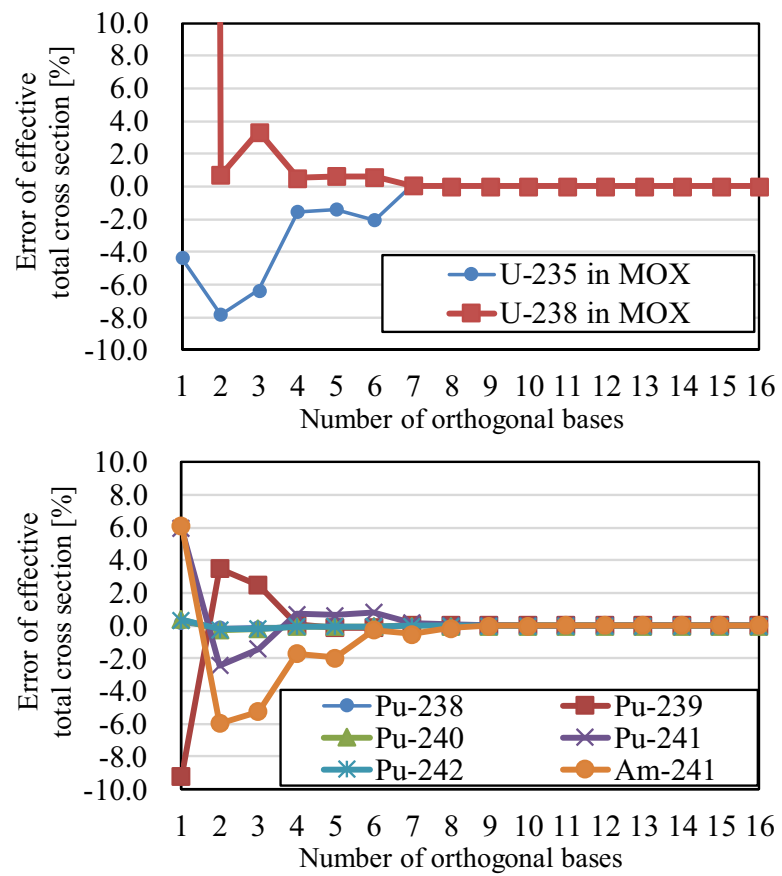

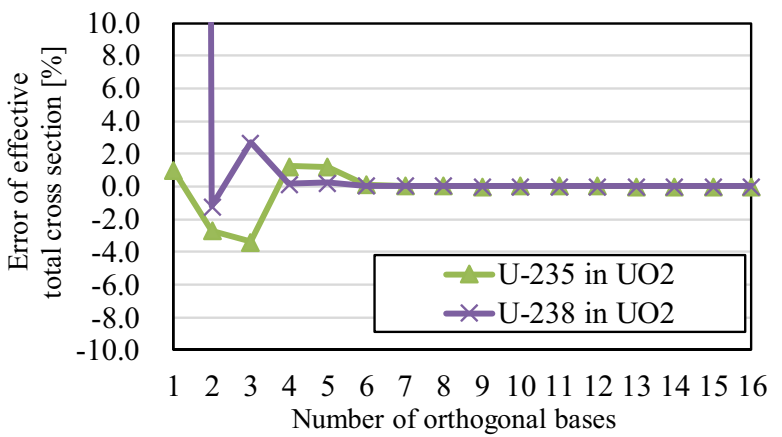

Figure 3. Comparison of effective total cross section in group 88 for various number of orthogonal bases in Fig. 1-(b).
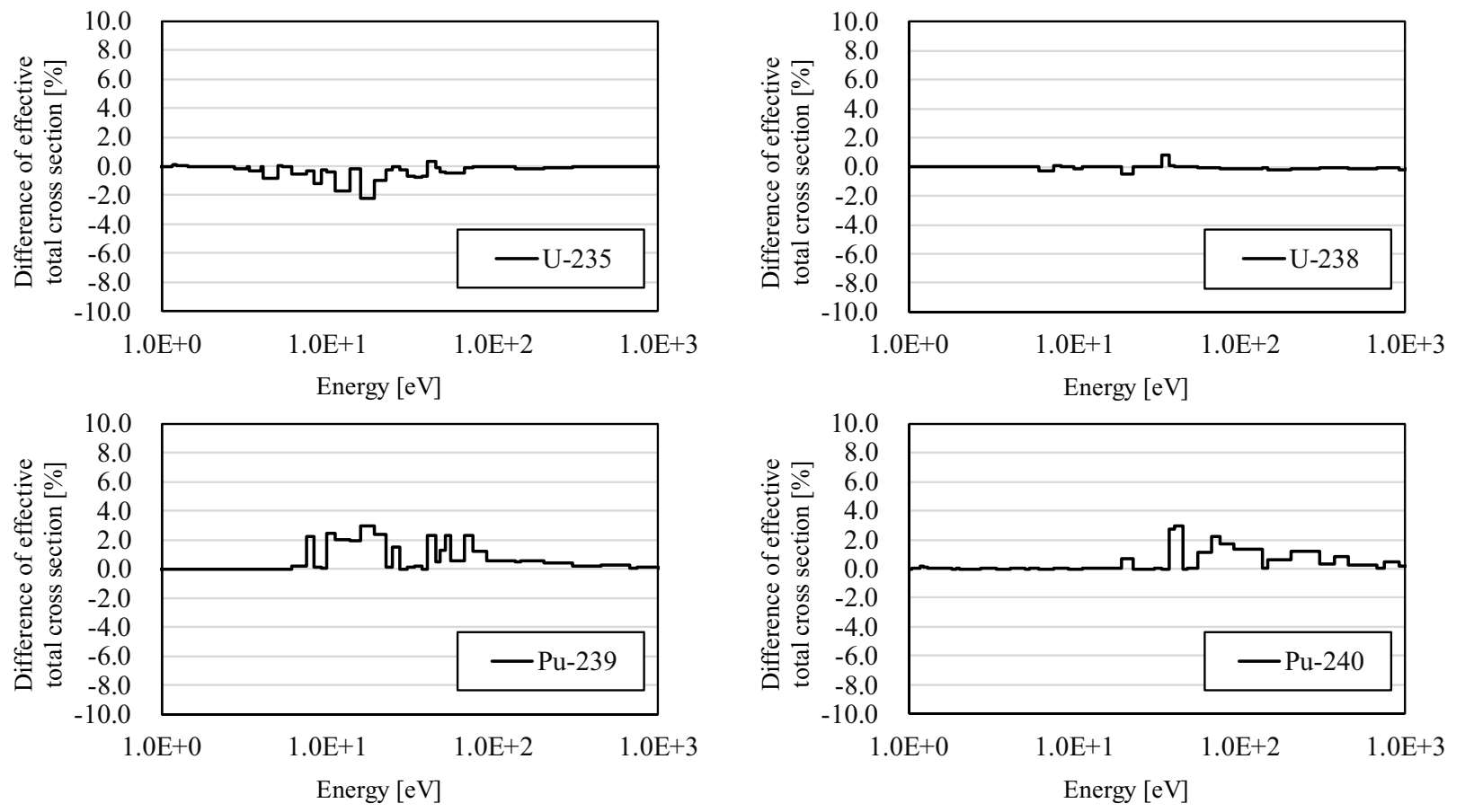

Figure 4. Difference of effective total cross sections in MOX between Fig. 1-(b) and Fig. 1-(c) obtained by UFG with MOC. 

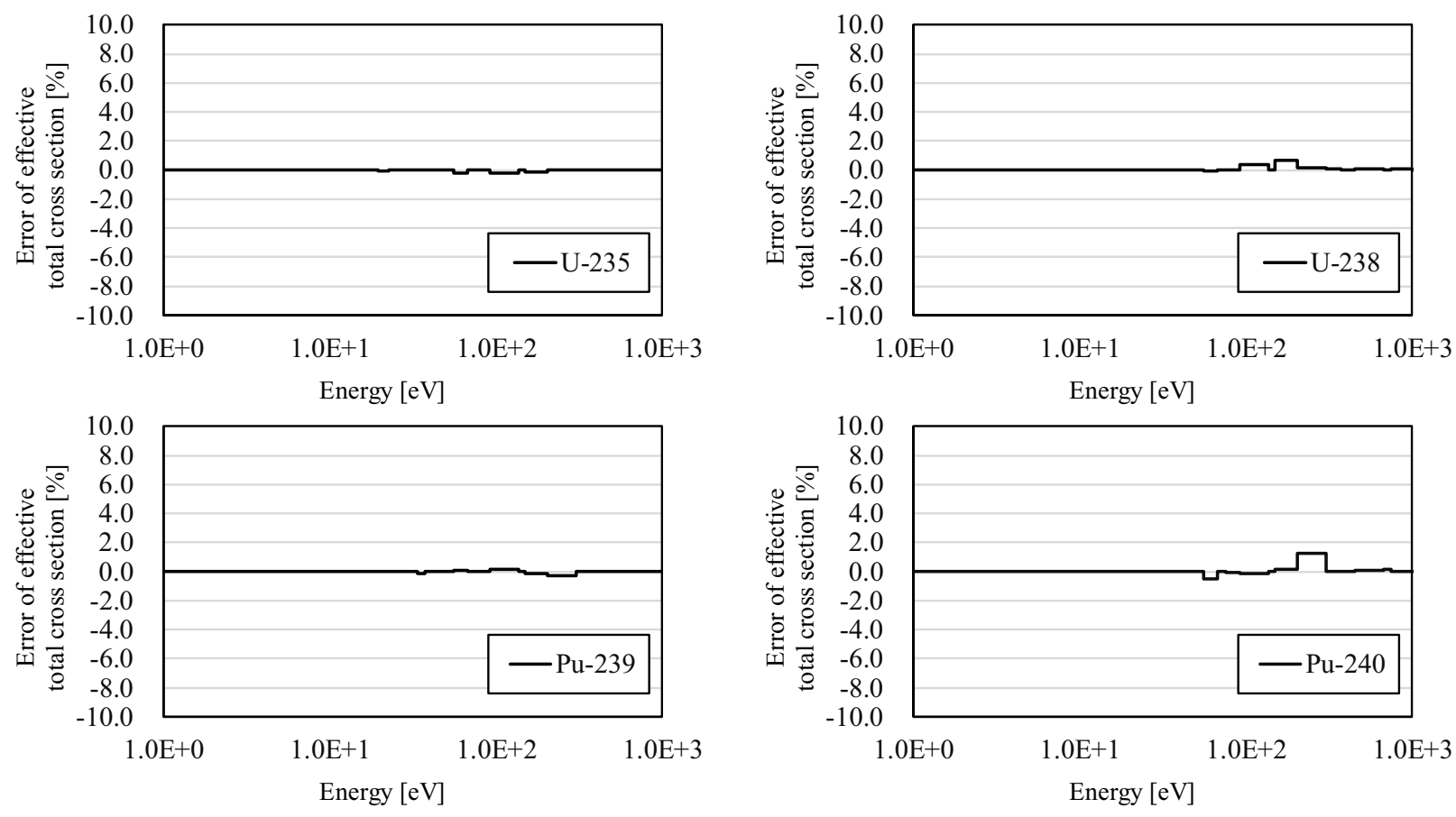

Figure 5. Error of effective total cross sections of U-235, U-238, Pu-239, and Pu-240 in MOX of Fig. 1-(b).
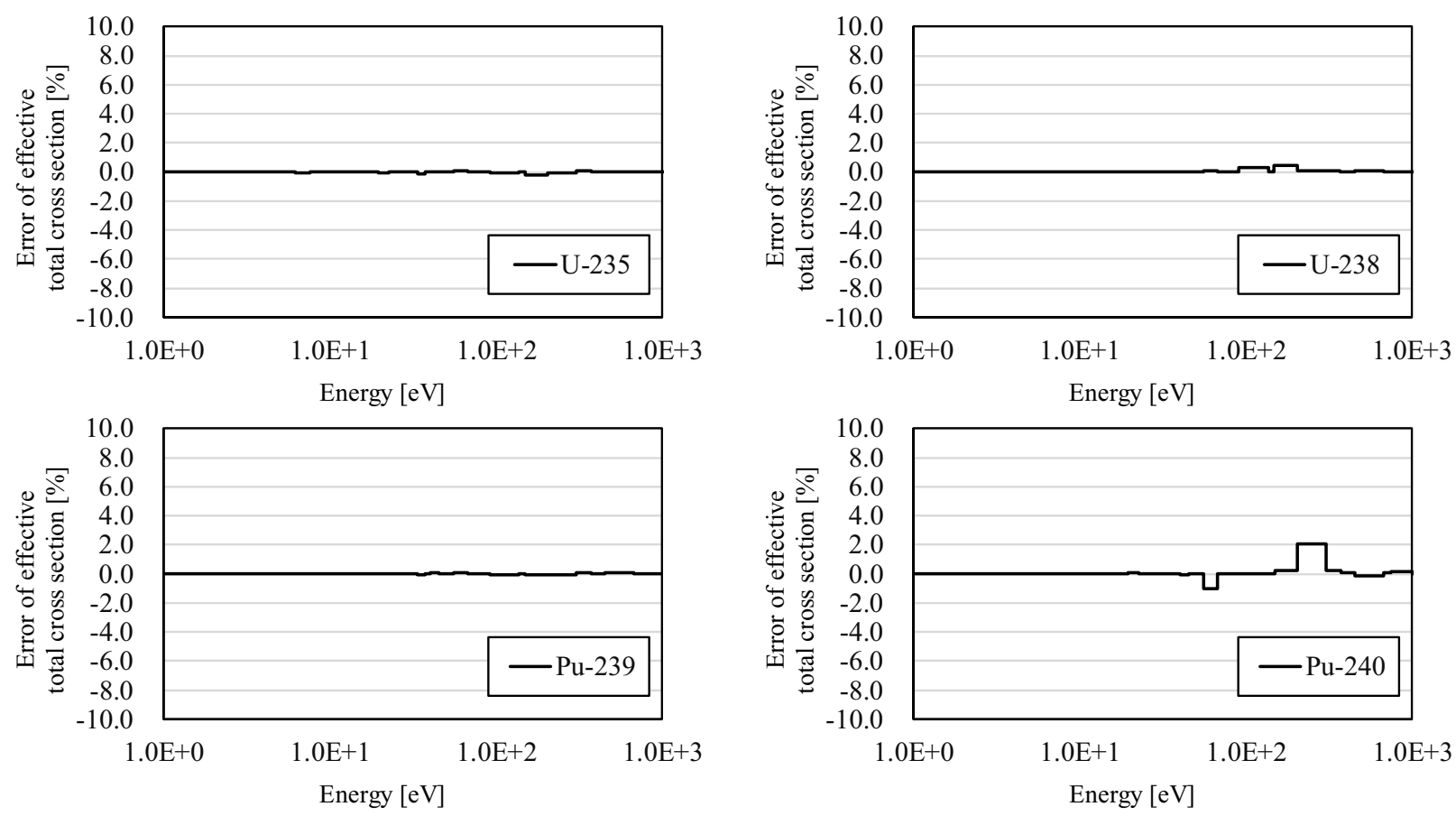

Figure 6. Error of effective total cross sections of U-235, U-238, Pu-239, and Pu-240 in MOX of Fig. 1-(c). 


\section{CONCLUSIONS}

The Resonance calculation based on energy Spectral Expansion (RSE) is applied to a $2 \times 2$ multi-cell geometries and its accuracy is confirmed through comparison with the results by the ultra-fine group heterogeneous calculation using MOC.

The numerical results show that the RSE method accurately reproduces the reference results obtained from the ultra-fine group calculation by MOC considering the resonance interference effect between different regions.

There are several issues to be addressed; 1) treatment of scattering source, 2) an efficient generation method of orthogonal basis, 3) application to more complicated conditions/large geometry, e.g., consideration of temperature distribution, fuel assembly or full core geometry 4) an efficient numerical calculation for matrix exponential, 5) estimation of computational cost for practical calculation conditions, and 6) comparison of calculation cost and accuracy with other methods, such as the equivalence theory, the ultra-fine group calculation, and the subgroup method.

\section{REFERENCES}

1. D. G. Cacuci ed., Handbook of Nuclear Engineering, Chap.9, Lattice Physics Computations, Springer, ISBN 978-0-387-98150-5, (2010).

2. A. Yamamoto et al., "A Resonance Calculation Method using energy Expansion Bases based on a Reduced Order Model," Proceedings of M\&C2019, Portland, OR, Aug. 25-29, 2019 pp. 1081-1092 (2019).

3. M. Matsushita, T. Endo, A. Yamamoto, T. Kitao, "Development of Reduced Order Model of Severe Accident Analysis Code for Probabilistic Safety Margin Analysis," Proceedings of PHYSOR2018, Cancun, Mexico, Apr. 22- Apr. 26, 2018, (2018). [USB].

4. R. Reed, J. Roberts, "Enhancements to the Discrete Generalized Multigroup Method," Trans. Am. Nucl. Soc., 120, 475 (2019).

5. F. Rahnema, S. Douglass, B. Forget, "Generalized Energy Condensation Theory," Nucl. Sci. Eng., 160, pp. 41 (2008).

6. L. Zhu B. Forget, "A Discrete Generalized Multigroup Energy Expansion Theory," Nucl. Sci. Eng., 166, pp. 239 (2010).

7. R. Le Tellier, D. Fournier, J. Ruggieri. "A Wavelet-based finite element method for the self-shielding issue in neutron transport." Nucl. Sci. Eng., 163, pp. 34 (2009).

8. W. Yang, H. Wu, Y. Zheng. "Application of wavelets scaling function expansion method in resonance self-shielding calculation," Ann. Nucl. Energy, 37, pp. 653 (2010).

9. W. Rooijen, "Feasibility of Wavelet Expansion Method to Treat the Energy Variable," Proceedings of PHYSOR2012, Knoxville, TN, Apr. 15-20, 2012 (2012). [USB]

10. R. E. Macfarlane, D. W. Muir, The NJOY Nuclear Data Processing System Version 91, LA-12740-M (October 1994).

11. K. Tada, Y. Nagaya, S. Kunieda, K. Suyama, T. Fukahori, "Development and verification of a new nuclear data processing system FRENDY," J. Nucl. Sci. Technol., 54, pp. 806-817 (2017).

12. K. Shibata, O. Iwamoto, T. Nakagawa et al., "JENDL-4.0: A New Library for Nuclear Science and Engineering," J. Nucl. Sci. Eng., 48, pp. 1-30 (2011).

13. A. Yamamoto, A. Giho, Y. Kato, T. Endo, "GENESIS - A Three-dimensional Heterogeneous Transport Solver based on the Legendre Polynomial Expansion of Angular Flux Method," Nucl. Sci. Eng., 186, 1 (2017).

14. A. Yamamoto, M. Tabuchi, N. Sugimura, T. Ushio, M. Mori, "Derivation of Optimum Polar Angle Quadrature Set for the Method of Characteristics Based on Approximation Error of the Bickley Function," J. Nucl. Sci. Technol., 44, pp.129 (2007). 